PROCEEDINGS OF THE

AMERICAN MATHEMATICAL SOCIETY

Volume 137, Number 3, March 2009, Pages 853-861

S 0002-9939(08)09713-X

Article electronically published on September 15, 2008

\title{
MAPS BETWEEN MODULI SPACES OF VECTOR BUNDLES AND THE BASE LOCUS OF THE THETA DIVISOR
}

\author{
TAWANDA GWENA AND MONTSERRAT TEIXIDOR I BIGAS
}

(Communicated by Ted Chinburg)

\begin{abstract}
We consider maps between different spaces of vector bundles on curves obtained by taking wedge powers, elementary transformations or kernels of evaluation maps and studying their respective fibers. We apply the results to construct large dimensional sets in the base locus of the generalized theta divisor.
\end{abstract}

\section{INTRODUCTION}

Given a vector bundle $E$ of rank $r$ and degree $d$ on a curve $C$ of genus $g \geq 2$, one can associate to $E$ in a natural way several other vector bundles. For example, one can take wedge powers of $E$. If $E$ is generated by global sections, the kernel of the evaluation map of sections is again a vector bundle. Also, new vector bundles can be produced by taking elementary transformations centered at a fixed point. Under suitable conditions on the degree and rank, these constructions can be carried out globally. While all these processes seem quite elementary, very little is known about the resulting maps. The purpose of this paper is to fill in this gap. We shall start by considering the kernel of the evaluation map of sections. If $E$ is generated by global sections, let $M_{E}$ denote the kernel of the evaluation map of sections of $E$. Then $M_{E}$ can be defined from the exact sequence

$$
0 \rightarrow M_{E} \rightarrow H^{0}(E) \otimes \mathcal{O}_{C} \rightarrow E \rightarrow 0 .
$$

Our main result is

1.1. Theorem. Let $d \geq 2 \mathrm{rg}$. Then the map between the moduli spaces of semistable vector bundles of rank $r$ and degree $d$ and rank $d-r g$ and degree $-d$ given by $E \rightarrow M_{E}$ is generically injective.

For the following result we assume that we are in characteristic zero. It is known then that the wedge powers of a semistable vector bundle are again semistable. Therefore, there is a rational map between the moduli space $U(r, d)$ of vector bundles of rank $r$ and degree $d$ and the moduli space of vector bundles of rank $\left(\begin{array}{l}r \\ i\end{array}\right)$ and degree $\left(\begin{array}{c}r \\ i\end{array}\right) i \frac{d}{r}$. We show

1.2. Theorem. Let $C$ be a projective non-singular curve defined over a field of characteristic zero. Let $i$ be an integer with $0<i<r$. Then the map between the

Received by the editors December 4, 2006, and, in revised form, January 23, 2008, and March 3, 2008.

2000 Mathematics Subject Classification. Primary 14H60.

(C) 2008 American Mathematical Society 
moduli space $U(r, d)$ and the moduli space $U\left(\left(\begin{array}{c}r \\ i\end{array}\right),\left(\begin{array}{c}r \\ i\end{array}\right) i \frac{d}{r}\right)$ given by $E \rightarrow \bigwedge^{i} E$ has finite fibers.

The same statement is true when replacing wedge powers by symmetric powers.

Finally consider elementary transformations. Starting with a vector bundle, one can produce another vector bundle of degree one more (or one less) that essentially differs from the original one only at one point. This construction can be done in many different ways and in general one can get an $r$-dimensional family of such transforms starting with a single vector bundle. We show here that if we start with a family of vector bundles and do all possible such transforms, the dimension of the vector bundles so obtained never goes down.

We then give an application in finding lower bounds on the dimension of the base locus of the theta divisor in the moduli space of vector bundles (see Theorem 5.2 and Corollary [5.3). For many values of the rank, this improves known results of Arcara $([\mathrm{A}])$, Popa $([\mathrm{P}])$ and Schneider $([\underline{\mathrm{S}}])$.

\section{InJeCtivity of The MAP $E \rightarrow M_{E}$}

If $E$ is a generic vector bundle of rank $r$ and degree at least $r g+1$, then it is generated by global sections. The same is true for every semistable vector bundle if the degree $d$ satisfies $d>r(2 g-1)$. Whenever $E$ is generated by global sections, one can define the vector bundle $M_{E}$ as in $\left(^{*}\right)$. Our goal in this section is to prove that the assignment $E \rightarrow M_{E}$ is generically injective when it is globally defined (namely $d \geq 2 r g+r)$. What we show in fact is a little bit more, namely that $M_{\left(M_{E}\right)^{*}}=E^{*}$. This in turn is equivalent to $h^{0}\left(\left(M_{E}\right)^{*}\right)=h^{0}(E)$. We shall reduce this statement to the surjectivity of a map between spaces of sections of vector bundles and prove the latter by induction on the rank.

Note that if $E$ is semistable of degree at least $2 \mathrm{rg}$, then $M_{E}$ is also semistable (see $[\mathrm{B}], 1.2)$.

2.1. Lemma. Let $C$ be a generic curve. Let $L$ be a line bundle on $C$ such that the complete linear system $L$ has no fixed points. Then, the map $C \rightarrow|L|$ is composed with an involution if and only if $L=\bar{L}^{2}$ with $\bar{L}$ a line bundle corresponding to a $g_{\frac{g+2}{2}}^{1}$ map on $C$.

Proof. Assume that the map associated to the line bundle $L$ is composed with an involution. As the curve $C$ is generic, it is not a covering of any curve of genus at least one. Therefore the map corresponding to the linear system $|L|$ factors through

$$
C \rightarrow C^{\prime} \rightarrow|L|
$$

where $C^{\prime}$ is a rational curve and the first map is given by a $g_{k}^{1}$. Because of the genericity of $C$, this implies that the corresponding Brill-Noether number $\rho=$ $g-2(g-k+1) \geq 0$ is positive. Equivalently, $k \geq \frac{g+2}{2}$. Moreover, $L=r g_{k}^{1}$ with $h^{0}(L)=r+1$.

Note now that $h^{1}\left(r g_{k}^{1}\right)=h^{0}\left(K-r g_{k}^{1}\right)=0$. This is because

$$
H^{0}\left(K-r g_{k}^{1}\right) \subset H^{0}\left(K-2 g_{k}^{1}\right) \subset \operatorname{Ker}\left(H^{0}\left(g_{k}^{1}\right) \otimes H^{0}\left(K-g_{k}^{1}\right) \rightarrow H^{0}(K)\right)
$$

and this kernel is zero by the injectivity of the Petri map for the generic curve.

Hence,

$$
r+1=h^{0}(L)=h^{0}\left(r g_{k}^{1}\right)=r k+1-g \geq r \frac{g+2}{2}+1-g \geq g+r+1-g=r+1 .
$$


Here the last inequality comes from the fact that $r \geq 2$. Hence, all inequalities must be equalities and then $r=2, k=\frac{g+2}{2}$ as stated.

2.2. Proposition. Let $L$ be a line bundle generated by global sections on a generic curve. Assume $L \neq \bar{L}^{2}$, where $\bar{L}$ is a line bundle corresponding to a $g_{\frac{g+2}{2}}^{1}$ map on C. Then, the map

$$
\psi: H^{0}(L) \otimes H^{0}(K) \rightarrow H^{0}(K \otimes L)
$$

is onto. The result is also true on any curve if $L$ is generic.

Proof. From Lemma 2.1, the map corresponding to the complete linear system $|L|$ is not composed with an involution and by assumption it has no fixed points. The same is true on any curve if $L$ is taken to be generic. By Riemann-Roch

$$
k=h^{0}(L)=d+1-g+h,
$$

where $h=h^{1}(L)=h^{0}\left(K \otimes L^{-1}\right)$. Choose $P_{1}, \ldots, P_{k-2}$ generic points on $C$. Then, $h^{0}\left(L\left(-P_{1}-\cdots-P_{k-2}\right)\right)=2, h^{0}\left(K \otimes L^{-1}\left(P_{1}+\cdots+P_{k-2}\right)\right)=h^{0}\left(K \otimes L^{-1}\right)=h$.

Moreover $L\left(-P_{1}-\cdots-P_{k-2}\right)$ has no fixed points and is therefore generated by global sections. Consider the map

$$
\bar{\psi}: H^{0}\left(L\left(-P_{1}-\cdots-P_{k-2}\right)\right) \otimes H^{0}(K) \rightarrow H^{0}\left(K \otimes L\left(-P_{1}-\cdots-P_{k-2}\right)\right) .
$$

By the base point free pencil trick, the kernel of $\bar{\psi}$ is $H^{0}\left(K \otimes L^{-1}\left(P_{1}+\cdots+P_{k-2}\right)\right)$ and by our choice this space has dimension $h$. Therefore, the image of the above map has dimension $2 g-h$.

Now, the $P_{i}$ have been chosen to impose independent conditions on the linear system $|L|$. Hence, they impose independent conditions on the image of the map $\psi$. Then,

$\operatorname{dim}(\operatorname{Im} \psi) \geq \operatorname{dim}(\operatorname{Im} \bar{\psi})+k-2=(2 g-h)+(d+1-g+h)-2=d+g-1=h^{0}(K \otimes L)$.

The next result is not needed in the following. For the sake of completeness, we show that the restriction in Proposition 2.2 is not arbitrary.

2.3. Lemma. Assume that $L=\bar{L}^{2}$, where $\bar{L}$ is a line bundle corresponding to a $g_{\frac{g+2}{2}}^{1}$ map on $C$. Then the morphism

$$
\psi: H^{0}(L) \otimes H^{0}(K) \rightarrow H^{0}(K \otimes L)
$$

is not onto.

Proof. Let $s_{0}, s_{1}$ be a basis of a section of $|\bar{L}|$. Then $s_{0}^{2}, s_{0} s_{1}, s_{1}^{2}$ are sections of $|L|$.

From the base-point-free pencil trick, the map

$$
\bar{\psi}:\left\langle s_{0}^{2}, s_{1}^{2}\right\rangle \otimes H^{0}(K) \rightarrow H^{0}(K \otimes L)
$$

has kernel $H^{0}\left(K \otimes L^{-1}\right)=H^{0}\left(K \otimes \bar{L}^{-2}\right)$, and as in the proof of Lemma 2.1, the latter is zero. Hence, the image has dimension $2 g$. As $h^{0}(K \otimes L)=2 g+1$, the map is not onto. In order to prove the claim, it suffices to show now that the image of the map $\psi$ is the same as the image of the map $\bar{\psi}$. This is equivalent to proving that the elements of the form $s_{0} s_{1} t, t \in H^{0}(K)$ are in the image of $\psi$.

We show first that the map

$$
\left\langle s_{0}, s_{1}\right\rangle \otimes H^{0}\left(K \otimes \bar{L}^{-1}\right) \rightarrow H^{0}(K)
$$


is onto. In fact, the kernel of this map is zero because this is a Petri map for a generic curve. Therefore the image has dimension

$$
2 h^{0}\left(K \otimes \bar{L}^{-1}\right)=2\left(h^{0}(\bar{L})+g-1-\operatorname{deg}(\bar{L})\right)=g
$$

as stated.

Then, given $t \in H^{0}(K)$, we can write

$$
t=a s_{0}+b s_{1}, a, b \in H^{0}\left(K \otimes \bar{L}^{-1}\right) .
$$

Then,

as needed.

$$
s_{0} s_{1} t=\left(a s_{1}\right) s_{0}^{2}+\left(b s_{0}\right) s_{1}^{2}
$$

2.4. Theorem. Let $C$ be a generic curve. Let $L$ be a line bundle on $C$ generated by global sections. Assume $L \neq \bar{L}^{2}$, where $\bar{L}$ is a line bundle corresponding to a $g_{\frac{g+2}{2}}^{1}$ map on $C$. Consider the vector bundle $M_{L}$ defined by the exact sequence

$$
0 \rightarrow M_{L} \rightarrow H^{0}(L) \otimes \mathcal{O}_{C} \rightarrow L \rightarrow 0 .
$$

Then, $h^{0}\left(\left(M_{L}\right)^{*}\right)=h^{0}(L)$. The same is true for every curve if $L$ is taken to be generic.

Proof. Dualizing the sequence above and taking global sections, one obtains

$$
0=H^{0}\left(L^{*}\right) \rightarrow H^{0}(L)^{*} \rightarrow H^{0}\left(\left(M_{L}\right)^{*}\right) \rightarrow H^{1}\left(L^{*}\right) \rightarrow H^{0}(L)^{*} \otimes H^{1}(\mathcal{O}) .
$$

Therefore, the statement of the proposition is equivalent to the injectivity of the map

$$
H^{1}\left(L^{*}\right) \rightarrow H^{0}(L)^{*} \otimes H^{1}(\mathcal{O}) .
$$

This in turn is the dual of the map

$$
\psi: H^{0}(L) \otimes H^{0}(K) \rightarrow H^{0}(K \otimes L),
$$

which has been proved to be onto in Proposition 2.2.

2.5. Proposition. Let $E$ be a generic vector bundle of rank $r$ and degree $d \geq$ $(2 r-1) g+1$. Then, the map

$$
\psi: H^{0}(E) \otimes H^{0}(K) \rightarrow H^{0}(K \otimes E)
$$

is onto.

Proof. By Lange's Conjecture (proved in [RT, Thms. 0.2 and 0.1), a generic vector bundle can be written as an extension of generic stable bundles

$$
0 \rightarrow E_{1} \rightarrow E \rightarrow E_{2} \rightarrow 0
$$

so long as the slopes satisfy

$$
\mu\left(E_{2}\right)-\mu\left(E_{1}\right) \geq g-1 .
$$

We prove the result by induction on the rank $r$. The case $r=1$ has been proved in Proposition 2.2. Assume now that $r \geq 2$ and write $E$ as an extension

$$
0 \rightarrow L \rightarrow E \rightarrow E_{2} \rightarrow 0,
$$

where $L$ is a generic line bundle of degree $g+1$ and $E_{2}$ is a generic vector bundle of degree at least $(2 r-2) g \geq(2(r-1)-1) g+1$. Then both $L$ and $E_{2}$ are generated by 
global sections and by the induction assumption the corresponding maps $\psi_{L}, \psi_{E_{2}}$ are onto. Consider the exact diagram

$$
\begin{aligned}
& 0 \quad \rightarrow \quad H^{0}(L) \otimes H^{0}(K) \quad \rightarrow \quad H^{0}(E) \otimes H^{0}(K) \quad \rightarrow \quad H^{0}\left(E_{2}\right) \otimes H^{0}(K) \quad \rightarrow \quad 0
\end{aligned}
$$

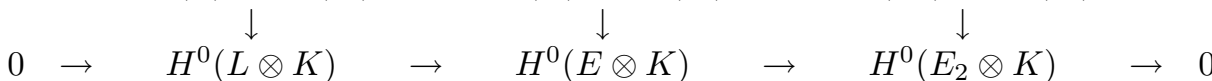

The zeros on the right are obtained because $h^{1}(L)=0$ as $L$ is generic of degree $g+1$ and also $h^{1}(K \otimes L)=0$.

As the left and right vertical maps are onto, so is the middle one.

2.6. Theorem. Let $C$ be a generic curve. Let $E$ be a generic semistable vector bundle on $C$ of degree at least $(2 r-1) g+1$. Consider the vector bundle $M_{E}$ defined by the exact sequence

$$
0 \rightarrow M_{E} \rightarrow H^{0}(E) \otimes \mathcal{O}_{C} \rightarrow E \rightarrow 0 .
$$

Then, $h^{0}\left(M_{E}^{*}\right)=h^{0}(E)$.

Proof. The proof is now identical to the one in the line bundle case, replacing Proposition 2.2 by Proposition 2.5 .

The proof of Theorem 1.1 immediately follows from the above as $E=M_{\left(M_{E}\right)^{*}}^{*}$. The condition $d \geq 2 \mathrm{rg}$ in Theorem 1.1 has been included in order to ensure that at the level of moduli spaces, the map is globally defined. It is likely though that $M_{E}$ is semistable for generic $E$ with the weaker condition $d \geq r g+1$.

\section{ELEMENTARY TRANSFORMATIONS}

Given a vector bundle $E$, a point $P$ and a linear map of the fiber $E_{P}$ of $E$ at $P$ to the base field $\mathbf{C}$, one has an exact sequence

$$
0 \rightarrow E^{\prime} \rightarrow E \rightarrow \mathbf{C}_{P} \rightarrow 0 .
$$

Then, $E^{\prime}$ is said to be obtained from $E$ by a (direct) elementary transformation.

Dualizing the above sequence, one obtains

$$
0 \rightarrow E^{*} \rightarrow E^{*} \rightarrow \mathbf{C}_{P} \rightarrow 0
$$

So, $E^{*}$ is obtained from $E^{\prime *}$ by a direct elementary transformation and $E$ is said to be obtained from $E^{\prime}$ by an inverse transformation.

3.1. Lemma. Let $C$ be a curve, $A$ a parameter space for a family of semistable vector bundles in $U(r, d)$. Let $B$ be a parameter space containing all bundles obtained from bundles in $A$ by doing an elementary transformation. Let

$$
f_{A}: A \rightarrow U(r, d), f_{B}: B \rightarrow U(r, d-1)
$$

be the rational maps induced by the families. Then $\operatorname{dim} f_{B}(B) \geq \operatorname{dim} f_{A}(A)$.

Proof. Assume that $\mathcal{E}$ is a vector bundle on $C \times A$ such that $\mathcal{E}_{\mid C \times\{a\}}=f_{A}(a)$. One can then construct a $B$ as follows: Denote by

$$
\pi_{12}: C \times A \times C \rightarrow C \times A, \pi_{13}: C \times A \times C \rightarrow C \times C
$$

the natural projections. Let $\triangle$ be the diagonal in $C \times C$. Take

$$
B=\mathbf{P}\left(\pi_{23 *}\left(\pi_{12}^{*}(\mathcal{E}) \otimes \pi_{13}^{*}\left(\mathcal{O}_{\triangle}\right)\right)\right) .
$$


It parameterizes triples

$$
(a, Q, s), a \in A, Q \in C, s: E_{a} \rightarrow \mathbf{C}_{Q}
$$

where $s$ is determined up to a scalar and $\mathbf{C}_{Q}$ is a one-dimensional skyscraper sheaf with support on $Q$.

Given $a \in A$, one obtains elements in $B$ by considering extensions of the form

$$
0 \rightarrow E^{*} \rightarrow E^{*} \rightarrow \mathbf{C}_{P} \rightarrow 0
$$

where $P \in C$ is a point. Equivalently, one has an exact sequence

$$
0 \rightarrow E^{*} \rightarrow E^{*} \rightarrow \mathbf{C}_{P} \rightarrow 0
$$

Given $E$, one can choose a point $P$ in $C$ and a map from the fiber of $E^{*}$ at $P$ to $\mathbf{C}_{P}$ up to multiplication with a constant. This then determines $E^{\prime}$. Assume that $\operatorname{dim} f_{B}(B)<\operatorname{dim} f_{A}(A)$. As every element in $A$ gives rise to an $r$-dimensional family of elements in $B$, this would imply that every element in $B$ comes from a family of elements in $A$ that is at least $(r+1)$-dimensional. From the first exact sequence a fixed $E^{\prime}$ in $B$ comes from a family of dimension at most $r$ in $A$. Hence, this is impossible.

\section{Symmetric AND WEDGE MAPS}

In this section, we need to work over a field of characteristic zero, as otherwise the wedge and symmetric powers of semistable bundles are not necessarily semistable. We prove Theorem 1.2 .

Proof. The proof that follows was suggested to the authors by S. Ramanan.

Note first that

$$
\operatorname{det}\left(\bigwedge^{i} E\right)=(\operatorname{det} E)^{\otimes\left(\begin{array}{c}
r-1 \\
i-1
\end{array}\right)}
$$

The map

$$
\begin{array}{cl}
\operatorname{Pic}^{d}(C) & \rightarrow \operatorname{Pic}^{d\left(\begin{array}{c}
r-1 \\
i-1
\end{array}\right)} \\
L & \left.\rightarrow L^{\otimes(} \begin{array}{c}
r-1 \\
i-1
\end{array}\right)
\end{array}
$$

is a finite map. Hence it suffices to prove the result for moduli spaces of vector bundles with fixed determinant.

The Picard group of the moduli spaces of vector bundles of given rank and determinant is $\mathbf{Z}$ generated by an ample divisor $\theta$ (see [DN]). Consider the map

$$
\begin{aligned}
& U(r, L) \rightarrow U\left(\left(\begin{array}{c}
r \\
i
\end{array}\right), L^{\left(\begin{array}{c}
r-1 \\
i-1
\end{array}\right)}\right) \\
& E \quad \rightarrow \quad \bigwedge^{\left(\begin{array}{l}
r \\
i
\end{array}\right)} E .
\end{aligned}
$$

It is easy to check that this is well defined too for equivalence classes of semistable bundles. This map is not constant. This can be proved for instance by deforming $E$ to a direct sum of line bundles for which the result is obvious.

The result follows now from the following (well-known) lemma:

4.1. Lemma. Let $f: X \rightarrow Y$ be a morphism of projective varieties with $\operatorname{Pic}(X)=$ Z. Then, either the map is constant or the fibers are finite.

Proof. Let $L$ be an element of $\operatorname{Pic}(Y)$ such that $f^{*}(L)$ is non-trivial. By the assumption on $\operatorname{Pic}(X)$, this implies that $f^{*}(L)$ is either ample or antiample. On the other hand, $f^{*}(L)$ is trivial on the fibers of $f$. Therefore, these fibers must be finite. 
The proof for symmetric powers is carried out in the same way.

\section{A LOWER BOUND FOR THE BASE LOCUS OF THE THETA DIVISOR IN MODULI SPACES OF VECTOR BUNDLES}

Denote by $U(r, L)$ the moduli space of vector bundles of rank $r$ and fixed determinant $L$ of degree $r(g-1)$. The Picard group of the moduli space is generated by the theta divisor that can be described as

$$
\left\{E \in U(r, L) \mid h^{0}(E) \geq 1\right\} .
$$

Consider the linear system in $U(r, L)$ associated to the theta divisor. Its base locus consists of those vector bundles $E$ such that $h^{0}\left(E \otimes L^{\prime}\right)>0$ for every $L^{\prime}$ of degree zero. It was proved first by Raynaud (see $[\mathrm{R}]$ ) that this base locus is non-empty. Later Popa showed in $[\mathrm{P}]$ that it is in fact of positive dimension. He used vector bundles of the form $\bigwedge^{\alpha} M_{L}$, where $L$ is a line bundle and $\alpha=\frac{g+1}{2}$ and suggested that maybe the $M_{E}$ for $E$ of rank higher than one might also work. We carry on this suggestion here. We need some preliminary results on properties of $M_{E}$ that are analogous to known properties for $M_{L}$ when $L$ is a line bundle.

5.1. Proposition (Compare with GL, section 2). Let $E$ be a generic vector bundle of rank $r$ and degree $d$. Let $M_{E}$ be the dual of the kernel of the evaluation map of $E$. If $j \leq d-r g-1$ and $P_{1}, \ldots, P_{j}$ are generic points on $C$, then there exists an exact sequence

$$
0 \rightarrow \mathcal{O}\left(P_{1}\right) \oplus \ldots \oplus \mathcal{O}\left(P_{j}\right) \rightarrow M_{E}^{*} \rightarrow F \rightarrow 0,
$$

where $F$ is a vector bundle of rank $d-r g-j$.

Proof. Choose generic points $P_{1}, \ldots, P_{j}$ and consider generic one-dimensional quotients $\mathbf{C}_{P_{1}}, \ldots \mathbf{C}_{P_{j}}$ of $E_{P_{1}}, \ldots, E_{P_{j}}$ respectively. Let $E_{1}$ be the elementary transform of $E$ associated to these quotients; namely, $E_{1}$ is defined by the exact sequence

$$
0 \rightarrow E_{1} \rightarrow E \rightarrow \mathbf{C}_{P_{1}} \oplus \ldots \oplus \mathbf{C}_{P_{j}} \rightarrow 0 .
$$

As $E$ is generic, so is $E_{1}$ (from section 3 ). By the assumption on the degree, it then follows that it is generated by global sections.

Let $V$ be the kernel of the map of vector spaces $H^{0}(E) \rightarrow \mathbf{C}_{P_{1}} \oplus \ldots \oplus \mathbf{C}_{P_{j}}$ and $W=H^{0}(E) / V$. Let $F^{*}$ be the kernel of the map $V \otimes \mathcal{O}_{C} \rightarrow E_{1}$. We then get a commutative diagram

$$
\begin{aligned}
& 0 \rightarrow F^{*} \rightarrow V \otimes \mathcal{O}_{C} \quad \rightarrow \quad \rightarrow \quad E_{1} \quad \rightarrow 0
\end{aligned}
$$

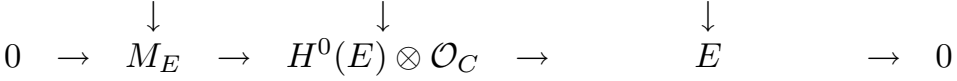

$$
\begin{aligned}
& 0 \rightarrow \stackrel{\downarrow}{A} \rightarrow \quad \rightarrow \stackrel{\downarrow}{\otimes} \mathcal{O}_{C} \rightarrow \mathbf{C}_{P_{1}} \oplus \cdots \oplus \mathbf{C}_{P_{j}} \rightarrow 0
\end{aligned}
$$

Here $A$ denotes the cokernel of the map $F^{*} \rightarrow M_{E}$. Then, from the lower exact row, $A=\mathcal{O}\left(-P_{1}\right) \oplus \cdots \oplus \mathcal{O}\left(-P_{j}\right)$. Dualizing the left exact sequence, the result follows.

5.2. Theorem. Let $r$ be an integer $r \geq 2$. Let $\beta=\operatorname{rg}(2 \mathrm{rg}-1)$ and let $L$ be a line bundle of degree $\beta(g-1)$. The theta linear system in the moduli space of vector bundles of rank $\beta$ and fixed determinant $L$ has a base locus of dimension at least $\left(r^{2}-1\right)(g-1)$. 
Proof. Let $L_{1}$ be a line bundle of degree $3 g$. Let $E$ be a vector bundle of rank $r$ and determinant $L_{1}^{r}$. Note that $E$ moves in a family of dimension $r^{2}(g-1)+1-g=$ $\left(r^{2}-1\right)(g-1)$. Then $M_{E}$ has rank $2 r g$ and $\operatorname{det}\left(\left(M_{E}\right)^{*}\right)=\operatorname{det}(E)=L_{1}$. Then

$$
\operatorname{det}\left(\bigwedge^{2}\left(\left(M_{E}\right)^{*}\right)\right)=\left(\operatorname{det}\left(M_{E}\right)^{*}\right)^{r_{M}-1}=\left(\operatorname{det}\left(M_{E}\right)^{*}\right)^{2 r g-1}=L_{1}^{(2 r g-1) r} .
$$

From our choices, the slope of

$$
\mu\left(\bigwedge^{2}\left(\left(M_{E}\right)^{*}\right)\right)=2 \mu\left(\left(M_{E}\right)^{*}\right)=2 \frac{3}{2}=3 .
$$

Let $P_{1}, \ldots, P_{g-2}, Q_{1}, Q_{2}$ be generic points on $C$. From Proposition 5.1 and the condition on $d, \mathcal{O}\left(Q_{1}\right) \oplus \mathcal{O}\left(Q_{2}\right)$ is a subsheaf of $\left(M_{E}\right)^{*}$. Hence $\mathcal{O}\left(Q_{1}+Q_{2}\right)$ is a subsheaf of $\bigwedge^{2} M_{E}$. Given a line bundle $T$ of degree $g-4$, there exist points $P_{1}, \cdots, P_{g-2}, Q_{1}, Q_{2}$ in $C$ such that $T=\mathcal{O}\left(P_{1}+\ldots+P_{g-2}-Q_{1}-Q_{2}\right)$. Hence,

$$
h^{0}\left(\bigwedge^{2}\left(M_{E}\right)^{*} \otimes T\right) \neq 0 .
$$

Let $L_{2}$ be a line bundle such that $L_{2}^{r g(2 r g-1)} L_{1}^{(2 r g-1) r}=L$. This implies that $L_{2}$ has degree $g-4$ and is determined up to a finite number of choices.

We claim that $\bigwedge^{2}\left(M_{E}\right)^{*} \otimes L_{2}$ is in the base locus of the theta divisor for $U(r g(2 r g-1), L)$. Note first that

$$
\begin{aligned}
\left.\operatorname{det}\left(\bigwedge^{2}\left(M_{E}\right)^{*} \otimes L_{2}\right)\right) & =L_{2}^{\operatorname{rank}\left(\bigwedge^{2}\left(M_{E}\right)^{*}\right)} \operatorname{det}\left(\bigwedge^{2}\left(M_{E}\right)^{*}\right) \\
& =L_{2}^{r g(2 r g-1)} L_{1}^{(2 r g-1) r}=L .
\end{aligned}
$$

Moreover, if $L_{0}$ is a generic line bundle of degree zero, then $L_{2} \otimes L_{0}=T$ is a generic line bundle of degree $g-4$. Hence, by the proof above,

$$
h^{0}\left(\bigwedge^{2}\left(M_{E}\right)^{*} \otimes L_{2} \otimes L_{0}\right) \neq 0,
$$

as needed.

Using Theorems 1.2 and 1.1 the dimension of the set of vector bundles $E$ and the dimension of the set of vector bundles $\bigwedge^{2}\left(M_{E}\right)^{*}$ are the same, so the claim follows.

5.3. Corollary. Let $j, \beta$ be defined as in the previous statement. If $\alpha>\beta$, then the moduli space of vector bundles of rank $\alpha$ and given determinant $L$ of degree $\alpha(g-1)$ has a base locus of dimension at least $\left(\left(r^{2}-1\right)+(\alpha-\beta)^{2}\right)(g-1)+1$.

Proof. Let $E_{1}$ be a vector bundle of rank $\alpha-\beta$ and slope $g-1$. Let $E_{2}$ be a vector bundle of rank $\beta$ and determinant $L \otimes \operatorname{det}\left(E_{1}\right)^{-1}$ in the base locus of the theta divisor. Then, $E=E_{1} \oplus E_{2}$ is in the base locus of the theta divisor as $h^{0}\left(E \otimes L_{0}\right) \geq h^{0}\left(E_{2} \otimes L_{0}\right)>0$ for every line bundle of degree zero. Hence, our claim follows from the previous theorem.

Remark. It was shown in [P] that the moduli space of vector bundles of rank $\left(\begin{array}{c}k(d-g) \\ \frac{g+1}{2}\end{array}\right)$ for any $k \geq 2$ has a base locus of dimension at least $(k-1) g$. Here we show that the moduli space of vector bundles of rank $r=\left(\begin{array}{c}2 t g \\ 2\end{array}\right)$ for any $t$ has a base locus of dimension at least $\left(t^{2}-1\right)(g-1)$. So our bound is basically linear on the rank. 
In $[\mathbf{S}$, lower bounds on the base locus of the theta divisor in $U(r, r(g-1))$ are obtained for $r \geq 2^{g}$, a bound that grows exponentially with $g$ while our bound on the rank is quadratic in $g$.

The best bounds for the dimension are obtained in [A], but the proof there is not constructive.

\section{REFERENCES}

[A] D. Arcara A lower bound for the dimension of the base locus of the generalized theta divisor, C. R. Acad. Sci Paris 340 (2005), 131-134. MR2116770 (2006b:14055)

[B] D. Butler Normal generation of vector bundles over a curve, J. Differential Geom. 39 (1994), no. 1, 1-34. MR1258911 (94k:14024)

[DN] J.M. Drezet, M.S. Narasimhan Groupe de Picard des variétés de modules de fibrés semistables sur les courbes algébriques, Invent. Math. 97 (1989), no. 1, 53-94. MR999313 (90d:14008)

[GL] M. Green, R. Lazarsfeld, A simple proof of Petri's theorem on canonical curves. Geometry today (Rome, 1984), 129-142, Progr. Math., 60, Birkhäuser Boston, Boston, MA, 1985. MR895152 (88h:14038)

[P] M. Popa, On the base locus of the generalized theta divisor, C. R. Acad. Sci. Paris Sér. I Math. 329 (1999), 507-512. MR 1715133 (2000j:14052)

[R] M. Raynaud, Sections des fibrés vectoriels sur une courbe, Bull. Soc. Math. France 110 (1982), 103-125. MR662131 (84a:14009)

[RT] B. Russo, M. Teixidor, On a conjecture of Lange, J. Alg. Geom 8 (1999), 483-496. MR:1689352(2000d:14039)

[S] O. Schneider, Sur la dimension de l'ensemble des points base du fibré déterminant sur $\mathcal{S} U_{C}(r)$, Ann. Inst. Fourier (Grenoble) 57 (2007), no. 2, 481-490. MR2310948 (2007m:14045)

Department of Mathematics, Tufts University, Medford, Massachusetts 02155

Current address: School of Mathematics, Georgia Institute of Technology, 686 Cherry Street, Skiles Building, Atlanta, Georgia 30332-0160

E-mail address: tgwena@gatech.edu

Department of Mathematics, Tufts University, Medford, Massachusetts 02155

E-mail address: montserrat.teixidoribigas@tufts.edu 